\title{
Los aliados emplumados de los Copales y Cuajiotes de México: aves y la dispersión de semillas de Bursera
}

\author{
Carlos A. Cultid-Medina y Yessica Rico
}

\begin{abstract}
Resumen
Los copales y cuajiotes, pertenecientes a la familia Burseraceae y del género Bursera, son árboles cuya diversidad de especies se concentra en México y son dominantes de los bosques tropicales secos. Tienen un alto valor cultural, económico y ecológico, ya que de ellos se extraen resinas aromáticas como el incienso, esencias naturales y madera para la elaboración de artesanías como alebrijes, máscaras y figurillas religiosas. A pesar de su importancia, el conocimiento de relaciones biológicas con especies asociadas a las burseras, como son sus polinizadores y dispersores de semillas, es aún limitado. En este trabajo se tratará la relación entre las aves y la burseras, al enfocarnos en cómo ocurre el proceso de la dispersión de semillas, la importancia que tiene para su supervivencia y las acciones concretas necesarias para la conservación de esta vital interacción.
\end{abstract}

Palabras clave: dispersión de semillas, Bursera, interacciones biológicas, aves, conservación.

\section{The feathered partners of Copales and Cuajiotes: Birds and the Bursera SEED DISPERSION}

\begin{abstract}
Copales and cuajiotes are trees, from the family Burseraceae and genus Bursera, whose center of species diversity is Mexico, being dominant species of the dry tropical forests. They have a high cultural, economic, and ecological value since they provide aromatic resins (incense), natural essences, and wood to produce crafts such as alebrijes, masks, and religious figures. Despite its importance, our knowledge on the biological relationships that exists between Bursera and their pollinators and seed dispersers is still limited. In this paper we discuss the relationship between birds and Burseras, we focus on explaining how the seed dispersal process occurs, the importance of such process for their survival and the concrete actions that are needed for the conservation of this vital interaction.
\end{abstract}

Keywords: seed dispersion, Bursera, biological interactions, birds, conservation.

Dol: http://doi.org/10.22201/codeic.16076079e.2020.v21n2.a5 


\section{Carlos A. Cultid-Medina ${ }^{1}$}

https://orcid.org/0000000249298405 carlos.cultid@inecol.mx

Investigador cátedra conacyt comisionado al Instituto de Ecología, A.C., Centro Regional del Bajío en Pátzcuaro, Michoacán. Sus línea de investigación abarcan, métodos para la evaluación multiescalar de la biodiversidad con énfasis en insectos indicadores de perturbación antrópica, interacciones biológicas y modelación estadística de datos ecológicos.

${ }^{1}$ ConAcyt, Av. Insurgentes Sur 1582, 03940, Ciudad de México, México.

Instituto de Ecología, A. C. Red de Diversidad Biológica del Occidente Mexicano. Centro Regional del Bajío, Av. Lázaro Cárdenas No. 253, Col. Centro, 61600 Pátzcuaro, Michoacán, México. +52 01 4341179521.

\section{Yessica Rico}

Doctora en Ecología y Biología Evolutiva por la Universidad de Toronto en Canadá. Investigadora cátedra conacyt comisionada al Instituto de Ecología A.C., Centro Regional del Bajío en Pátzcuaro, Michoacán. Sus líneas de investigación abarcan la genética de poblaciones, ecología, y biología de la conservación, principalmente en especies arbóreas, entre las que se encuentran las burseras.

${ }^{2}$ CONACYT, Av. Insurgentes Sur 1582, 03940, Ciudad de México, México.

Instituto de Ecología, A. C. Red de Diversidad Biológica del Occidente Mexicano. Centro Regional del Bajío, Av. Lázaro Cárdenas No. 253, Col. Centro, 61600 Pátzcuaro, Michoacán, México. +52 01 4341179524. 


\section{Introducción}

Seguramente en algún momento te has preguntado ¿cómo es que las plantas están en todas partes si no se mueven? Aunque no se desplazan como los animales, las plantas se mueven a través de las semillas, ya que durante su larga historia evolutiva se han seleccionado estrategias extraordinarias para estar a lo largo y ancho del planeta, al usar agentes vivos (bióticos) y no vivos (abióticos) (Murray, 2012).

Hay plantas que en sus semillas tienen estructuras aladas (ej. semilla de la jacaranda) que les permiten aprovechar el viento para ser transportadas lejos, mientras que otras poseen espinas o sustancias pegajosas que facilitan su adhesión al pelo de los animales. Pero ¿para qué sirven estas estrategias? En las plantas con flores (angiospermas) todas éstas tienen el mismo objetivo: garantizar que las semillas se alejen lo más lejos posible de sus padres y de sus semillas hermanas, evitando así la reproducción con los individuos de su familia mientras promueven la ocupación de nuevos sitios. Los científicos Ilaman este fenómeno dispersión (Dalling, 2002) y es el proceso biológico más importante para la supervivencia de plantas después de la polinización, debido a que, incluso si hay polinización, si se carecen de los medios para la dispersión de sus semillas, las plantas estarán en riesgo de desaparecer.

A causa de su variedad, los científicos clasifican las estrategias de dispersión según el agente involucrado. Por ejemplo, existen plantas que se dispersan por el esfuerzo explosivo de sus semillas (autocoria), a través del agua (hidrocoria), usando el viento (anemocoria) o buscando aliados en los animales (zoocoria) (Murray, 2012). En este último caso, encontramos formas variadas y llamativas en que las plantas se relacionan con diferentes tipos de animales, como las aves frugívoras que se encargan de comer frutos y semillas.

Aunque la zoocoria es una de las estrategias de dispersión más común entre las plantas con flor (Dalling, 2002), la interacción entre planta y ave es un fenómeno que apenas empezamos a entender para muchas especies de flora. Un ejemplo de esto es la relación entre las aves y los árboles conocidos popularmente como copales y cuajiotes. A continuación, explicaremos algunos aspectos sobre la reproducción y dispersión de semillas de estos árboles y abordaremos la importancia que tienen las aves para la supervivencia de los bosques tropicales secos.

\section{Una flora muy mexicana}

Pertenecientes al género botánico Bursera, los copales y cuajiotes son árboles o arbustos que pierden sus hojas durante la época seca del año, o sea, son caducifolios. En México existe la mayor diversidad de especies de burseras, con alrededor de 90 especies, la mayoría exclusivas del país (Rzedowski et al., 2005). 
Estos árboles evolucionaron a la par de los bosques tropicales secos del occidente de México. Son de gran importancia cultural y religiosa desde la época prehispánica, ya que durante este perío do se usaban en la elaboración de incienso para ceremonias religiosas por su cualidad de producir resinas aromáticas de olores intensos y agradables (López, 2016). De hecho, la palabra copal, proviene del náhuatl copalli, que significa incienso. Por su parte, los cuajiotes se distinguen de los copales por presentar una corteza exfoliante, parecida a escamas (cuajiote deriva del náhuatl quauihxiotl, árbol leproso). Además de la elaboración de incienso, la madera de los copales se emplea para la fabricación de artesanías: los alebrijes en Oaxaca o las máscaras en Michoacán; mientras que a los cuajiotes se les atribuyen propiedades medicinales. Desafortunadamente, varias especies son vulnerables a la extinción debido al desmonte de los bosques tropicales secos para la expansión de ciudades, zonas agropecuarias y la extracción de madera para obtener leña y hacer construcciones.

\section{¿Qué o quién dispersa las semillas de las burseras?}

Los dispersores son variados (coyotes, ratones, tejones y primates), pero sus principales aliados son las aves (Greenberg et al., 1995; Stevenson et al., 2005). A pesar de la importancia de las burseras, apenas estamos empezando a conocer qué aves ayudan a la dispersión de sus semillas.

Hoy sabemos que más de una decena de especies ayudan a las burseras a moverse hacia otros sitios y, posteriormente, en su germinación (ver figura 1). Ejemplos de estas aves son dos especies endémicas, el Carpintero del Balsas (Melanerpes hypopolius) y el Carpitero enmascarado (Melanerpes chrysogenys); otras especies como el Papamoscas gritón (Myiarchus tyrannulus) se encuentran a lo largo del continente americano o como la Calandria de Baltimore (Icterus galbula), con una distribución más restringida en Norteamérica. Algunas aves son especies migratorias que vienen del norte y se dirigen a los bosques tropicales secos del sur durante el invierno, por ejemplo, el Vireo ojos blancos (Vireo griseus), convirtiendo el fruto de las burseras en un importante recurso alimenticio. 


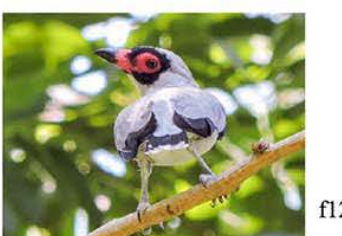

Titira Puerquito

(Tityra semifasciata)

f1

Vireo Ojos - Blancos

(Vireo griseus)

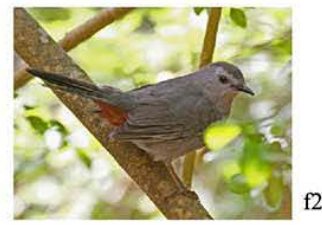

Maullador Gris (Dumetella carolinensis)

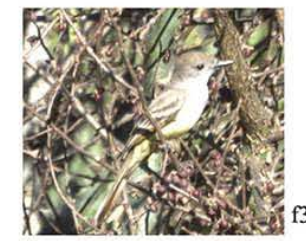

Papamoscas Cenizo (Myiarchus cinerascens)

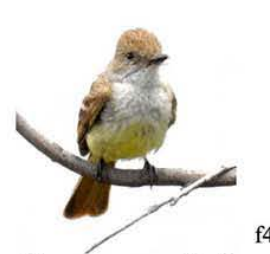

Papamoscas Huí (Myiarchus nuttingi)

4

Greenberg et al., 1995

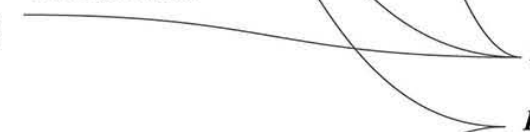

B. simaruba

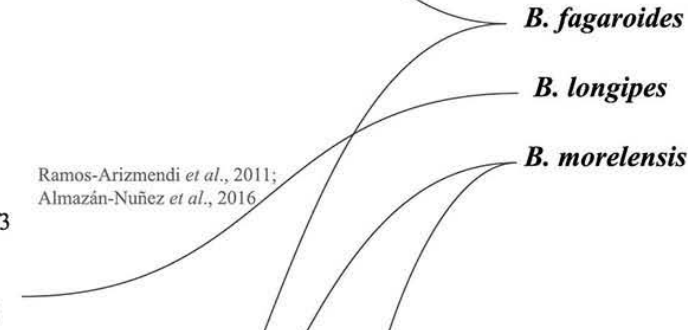

Ramos-Arizmendi et al., 2011

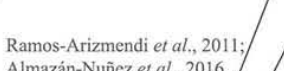

Almazán-Nuñez et al., 2011 .

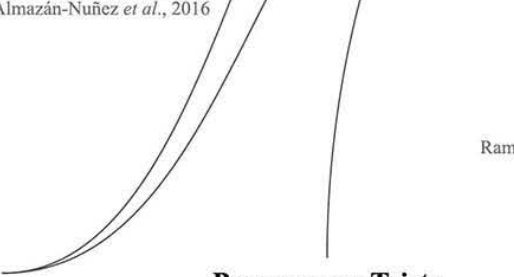

Papamoscas Triste

(Myiarchus tuberculifer)

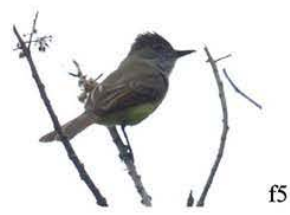

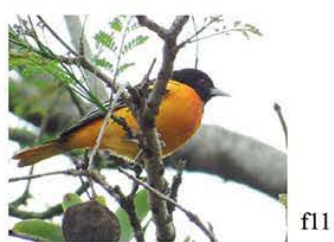

Calandría de Baltimore (Icterus galbula)

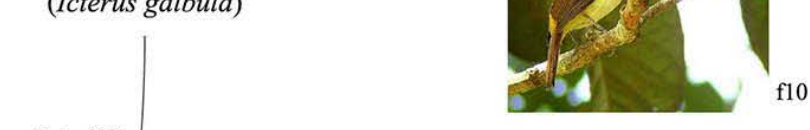

Ramos-Arizmendi et al., 2011

Calandria Dorso Rayado (Icterus pustulatus)

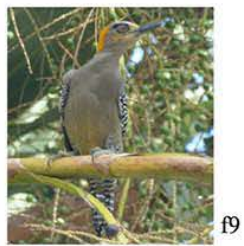

Almazán-Nuñez et al.,2016

Carpintero Enmascarado (Melanerpes chrysogenys ${ }^{*}$ )

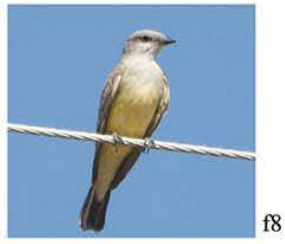

Tirano Pálido (Tyrannus verticalis)

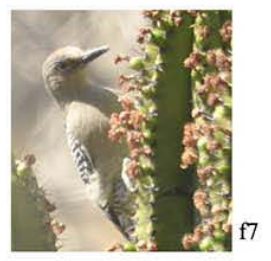

Carpintero del Balsas (Melanerpes hypopolius*)
Figura 1. Algunas de las especies de aves que contribuyen a la dispersión de semillas de algunas especies de copales y cuajiotes (Bursera spp.). Se muestra el nombre común y el nombre científico de las aves. * Especies Endémicas de México. 


\section{¿Cómo se da el proceso de dispersión?}

Para garantizar la dispersión de sus semillas, las burseras presentan frutos ovoides (drupas) con cubiertas relativamente duras, que al madurar se abren y exponen la semilla, que, a su vez, está rodeada por una estructura muy colorida, roja, naranja o amarilla, llamada pseudoarilo (Rzedowski y GuevaraFérer, 1992). Su función es atraer la atención de las aves para que consuman la semilla. Una vez que éstas ingieren el fruto completo o la semilla expuesta con el pseudoarilo, reciben como recompensa lípidos, proteínas, y azúcares, que les brindan energía y nutrientes básicos (Greenberg et al., 1995). Dentro del sistema digestivo del ave, debido a la acción de los ácidos gástricos, la semilla queda expuesta y su dura cubierta (pireno) es parcialmente disuelta y se ablanda, lo que facilita su germinación una vez que sea excretada. Todo este proceso se llama escarificación (ver figura 2).

Una peculiaridad de algunas especies de burseras es la producción de frutos sin semillas a partir de flores no polinizadas (Ramos-Ordoñez y Arizmendi, 2011). Esto tiene una doble función, por un lado, reduce el efecto de la depredación de los frutos viables (semillas consumidas y destruidas) y, por el otro, incrementa el éxito de la dispersión por parte de dispersores más efectivos, es decir, aquellas aves que logran esparcir las semillas a sitios óptimos y alejados de la planta madre (Ramos-Ordoñez y Arizmendi, 2011). Sin embargo, la producción de frutos sin semilla se puede deber a otras causas, como defectos genéticos o por el ataque de insectos que llevan al aborto del óvulo.

\section{¿Qué tan lejos son llevadas las semillas?}

Las aves aliadas de las burseras se pueden desplazar cientos y hasta miles de metros en pocos días, por lo tanto, las semillas pueden ser llevadas muy lejos de sus árboles madre. Esto explica en parte cómo las plantas están presentes a través de extensas áreas. No obstante, el éxito de la dispersión no sólo depende de la distancia que recorran las aves, sino también de que la semilla sea depositada en lugares de buena calidad para su germinación y desarrollo. Para ello, las semillas requieren condiciones óptimas de suelo, lo que depende del tipo de especie. De igual manera, las plantas jóvenes necesitan la protección de otras plantas para desarrollarse hasta que alcancen cierta altura e incrementen el grosor de su tronco. Las plantas protectoras son llamadas nodrizas y ofrecen sombra protectora contra los rayos del sol, así como un sustrato con mayor humedad y nutrientes (ver figura 2). 


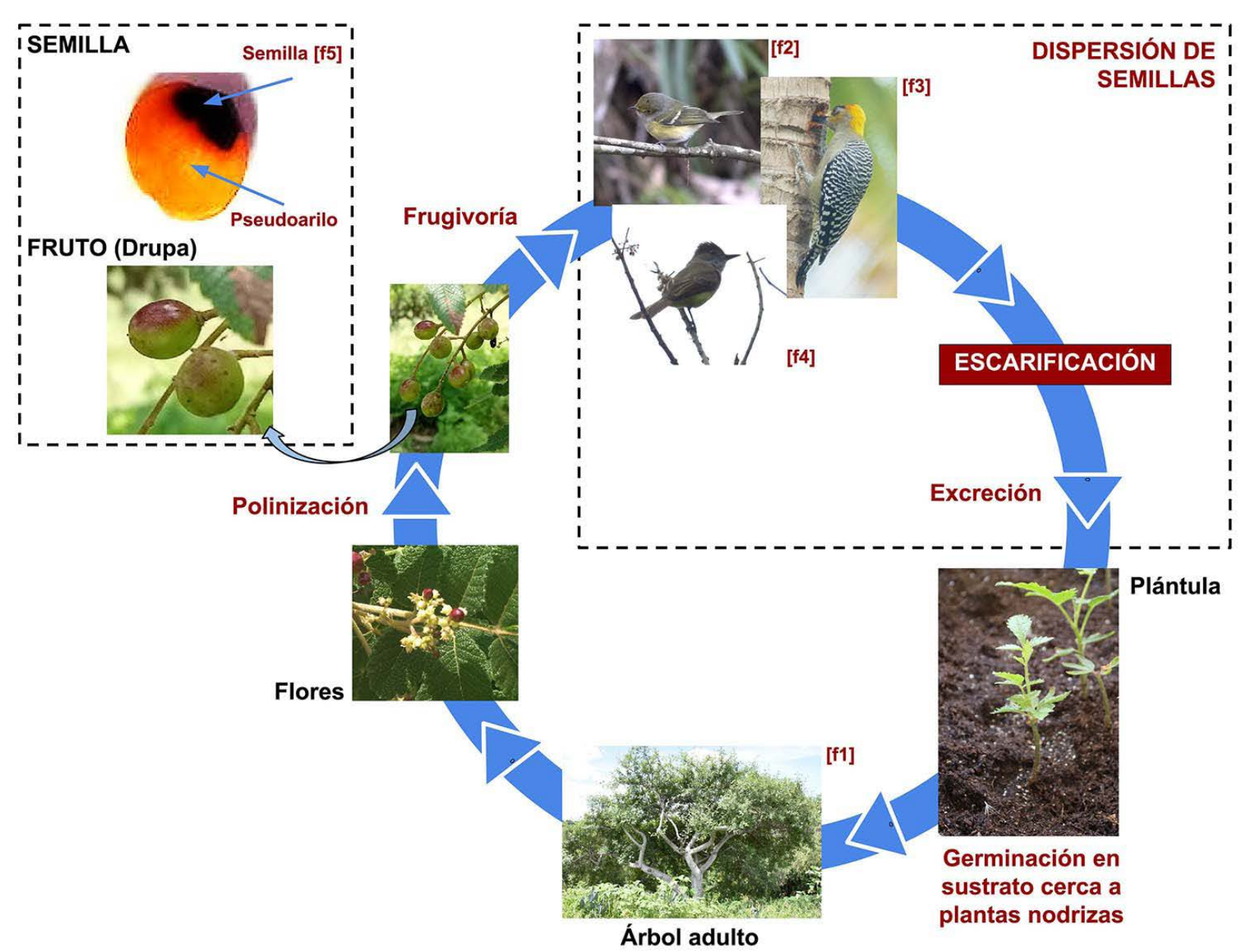

Figura 2. Las aves y la dispersión de las semillas de los copales y cuajiotes.

f1. Bursera cuneata (Copal). Fotografía de Rico, Y., 2019.

f2. Vireo griseus (Vireo Ojos Blancos).

Fotografía de Marín, O., 2016.

f3. Melanerpes chrysogenys (Carpintero Enmascarado). Fotografía de Marín, O., 2018.

f4. Myiarchus tuberculifer (Papamoscas Triste). Fotografía de Marín, O. 2019.

\section{¿Cómo cuidar la alianza entre las aves y las burseras?}

Más allá de ofrecer alimento a las aves, las burseras tienen un papel central en amplias redes de interacciones biológicas, que apenas se están dando a conocer. Son árboles dominantes de los bosques tropicales secos y ofrecen una variedad de servicios relacionados con el ecosistema que benefician al ser humano, como la regulación de la temperatura, el reciclaje de nutrientes, la captación del agua y la protección contra la erosión del suelo. Por lo tanto, todos podemos ayudar a mantener la relación entre las burseras y las aves si trabajamos en mejorar los siguientes puntos:

1. Garantizar la conservación y manejo sustentable de áreas conservadas de bosque tropical seco donde viven las burseras en México.

2. Promover un crecimiento ordenado y consensuado de nuestras áreas agropecuarias y urbanas. Esto no sólo protegerá los hábitats de las burseras, sino también el hábitat de las aves que ayudan a la dispersión de sus semillas, y de otras especies de flora. 
"Los aliados emplumados de los Copales y Cuajiotes de México: aves y la dispersión de semillas de Bursera"

Carlos A. Cultid-Medina y Yessica Rico

Vol. 21, Núm. 2, marzo-abril 2020

Revista Digital Universitaria

3. Diseñar e implementar programas comunitarios para la propagación de especies nativas de copales y cuajiotes, permitiendo la restauración de los bosques y su uso sustentable.

4. Realizar actividades de divulgación científica para comunicar la importancia ecológica de las burseras.

5. Incrementar el apoyo para la investigación científica y participativa sobre el papel ecológico de la flora arbórea de México.

\section{Referencias}

* Almazán-Núñez, R. C., Eguiarte, L. E., del Coro Arizmendi, M., \& Corcuera, P. (2016). Myiarchus flycatchers are the primary seed dispersers of Bursera longipes in a Mexican dry forest. Peer], 4, e2126. Dol: https://doi.org/10.7717/peerj.2126.

* Art Mur (s.f.). Papamoscas Huí (Myiarchus nuttingi) [fotografía]. iNaturalist. Recuperado de: http://www.inaturalist.org/photos/4045170.

* Dalling, J. W. (2002). Ecología de semillas. Ecología y conservación de bosques neotropicales, 345-375.

* Foster, M. S. (2007). The potential of fruit trees to enhance converted habitats for migrating birds in southern Mexico. Bird Conservation International, 17(1), 45-61. Dol: https://doi.org/10.1017/S0959270906000554.

Greenberg, R., Foster, M. S., y Marquez-Valdelamar, L. (1995). The role of the whiteeyed vireo in the dispersal of Bursera fruit on the Yucatan Peninsula. Journal of Tropical Ecology, 11(4), 619-639. Dol: https://doi.org/10.1017/S0266467400009184.

* Greglasley (s.f.). Papamoscas Gritón (Myiarchus tyrannulus) [fotografía]. iNaturalist. Recuperado de: http://www.inaturalist.org/photos/223067.

* Harleston, Cheryl. (s.f.). Calandria Dorso Rayado (Icterus pustulatus) [fotografía]. iNaturalist. Recuperado de: https://www.naturalista.mx/photos/1537397.

- Harleston, Cheryl. (s.f.). Titira Puerquito (Tityra semifasciata) [fotografía]. iNaturalist. Recuperado de: http://www.inaturalist.org/photos/2251278.

* jalinage00 (s.f.). Vireo Ojos Blancos (Vireo griseus) [fotografía]. iNaturalist. Recuperado de: https://www.naturalista.mx/taxa/17408-Vireo-griseus.

* López, A. (2016). Copal de Bursera bipinnata. Una resina mesoamericana de uso ritual. Trace, 70, 45-78. Recuperado de: https://journals.openedition.org/ trace/2274.

* Marín, Oscar H. (s.f.). Calandria de Baltimore (Icterus gálbula) [fotografía]. iNaturalist. Recuperado de: https://www.inaturalist.org/photos/29473748.

* Marín, Oscar H. (s.f.). Carpintero del Balsas (Melanerpes hypopolius) [fotografía]. iNaturalist. Recuperado de: https://www.inaturalist.org/photos/29385174.

* Marín, Oscar H. (s.f.). Carpintero Enmascarado (Melanerpes chrysogenys) [fotografía]. iNaturalist. Recuperado de: https://www.inaturalist.org/photos/29209736. 
"Los aliados emplumados de los Copales y Cuajiotes de México: aves y la dispersión de semillas de Bursera"

Carlos A. Cultid-Medina y Yessica Rico

Vol. 21, Núm. 2, marzo-abril 2020

Revista Digital Universitaria

* Marín, Oscar H. (s.f.). Papamoscas Cenizo (Myiarchus cinerascens) [fotografía]. iNaturalist. Recuperado de: https://www.inaturalist.org/photos/29385106.

- Marín, Oscar H. (s.f.). Papamoscas Triste (Myiarchus tuberculifer) [fotografía]. iNaturalist. Recuperado de: https://www.inaturalist.org/photos/37511745.

* Marín, Oscar H. (s.f.). Tirano Pálido (Tyrannus verticalis) [fotografía]. iNaturalist. Recuperado de: https://www.inaturalist.org/photos/29385205.

- Murray, D. R. (Ed.). (2012). Seed dispersal. Academic Press.

- Oldenette, Jerry (s.f.). Maullador Gris (Dumetella carolinensis) [fotografía]. iNaturalist. Recuperado de: https://www.naturalista.mx/taxa/14995-Dumetella-carolinensis.

* Ortiz-Pulido, R., \& Rico-Gray, V. (2006). Seed dispersal of Bursera fagaroides (Burseraceae): the effect of linking environmental factors. The Southwestern Naturalist, 51(1), 11-22. Dol: https://doi.org/10.1894/0038-4909(2006)51[11:SDOBF B]2.0.CO;2.

* Ramos-Ordoñez, M. F., y Arizmendi, M. C. (2011). Parthenocarpy, attractiveness and seed predation by birds in Bursera morelensis. Journal of Arid Environments, 75(9), 757-762. Dol: https://doi.org/10.1016/j.jaridenv.2011.04.013.

- Rzedowski, J., y Guevara-Férer, F. (1992). Burseraceae. Flora del Bajío y de regiones adyacentes, fascículo 3: 1-46. Recuperado de: http://inecolbajio.inecol.mx/ floradelbajio/documentos/fasciculos/ordinarios/Burseraceae\%203.pdf.

* Rzedowski, J., Medina, R. y Calderón, G. (2005). Inventario del conocimiento taxonómico, así como de la diversidad y del endemismo regionales de las especies mexicanas de Bursera (Burseraceae). Acta Botánica Mexicana, 70: 85-111. Dol: https://doi.org/10.21829/abm70.2005.989.

- Stevenson, P., Link, A. y Ramírez, B. (2005). Frugivoryand Seed Fate in Bursera inversa (Burseraceae) at Tinigua Park, Colombia: Implications for Primate Conservation. Biotropica, 37, 431-438. Dol: https://doi.org/10.1111/j.1744-7429.2005.00057.x

\section{Agradecimientos}

Al Consejo de Ciencia y Tecnología (CONACrT) por el financiamiento otorgado al proyecto de ciencia básica CB-2016-283237.

\section{Cómo citar este artículo}

- Cultid-Medina, Carlos A. y Rico, Yessica (2020). Los aliados emplumados de los copales y cuajiotes de México: aves y la dispersión de semillas de Bursera. Revista Digital Universitaria (RDU). Vol. 21, núm. 2 marzo-abril. Dol: http://doi.org/10.22201/ codeic.16076079e.2020.v21n2.a5.

Recepción: 11/05/2019. Aprobación: 04/02/2020 